\title{
Impacto de las aulas de creación en el nivel medio superior: el caso
}

\author{
Workshops' artistic creation impacts: the case ITESO
}

Javier GONZÁLEZ. Universidad de Guanajuato (México)

jr2000x@yahoo.es,jr2000x@gmail.com

Resumen: El artículo plantea un análisis etnográfico del impacto de aulas y talleres de creación artística en Educación Media y Superior. Para ello se analiza en profundidad el caso de la red de aulas y talleres artísticos de Promoción Cultural del ITESO, a través de la revisión de un proyecto de investigación que recuperó la experiencia vivida, sirviendo de retroalimentación para la propia institución. En el desarrollo de esta investigación se apunta a una serie de criterios, ya detectados en distintas líneas de investigación, que pudieran favorecer un "enfoque proactivo de la creatividad", confirmar la figura del artista-pedagogo, poco conocida en el contexto universitario, y un tipo de didáctica que se ha denominado "apreciativa y/o generativa" centrada en la formación de actitudes. Esta experiencia pudiera servir de referente para otras instituciones, a la hora de abrir espacios en forma de redes de conocimiento grupal y afectivo más allá de la educación formal, donde cada institución pudiera construir sus respuestas en función de sus necesidades y contextos. Hemos asistido a un proceso de desarrollo. Se abre el despertar emocional de la creación como proceso evolutivo, donde las ideas se convierten en auténticas vivencias.

Palabras clave: Taller, Creación Artística, Aprendizaje, Desarrollo Evolutivo, Educación Superior.

Abstract: This article presents an ethnographic analysis of the impact of classrooms
and workshops of artistic creation and Higher Education. For it is analyzed in
depth the case of the network of classrooms and art workshops Cultural Promotion
ITESO, through the review of a research project that recovered the experience,
serving as feedback for the institution itself. In the development of this research 
points to a number of criteria, and identified different lines of research that might favor a "proactive approach to creativity," confirm the figure of the artist-educator, little known in the university context, and a type of teaching that has been called "appreciative and / or generative" focused on shaping attitudes. This experience could serve as a model for other institutions, when it comes to open spaces in the form of group and affective networks beyond formal education, where each institution could build their responses according to their needs and knowledge contexts. We have witnessed a process of development. The emotional awakening of creation as an evolutionary process, where ideas become real experiences opens.

Keywords: Workshop, Artistic Creation, Learning, Evolutionary Development, Higher Education

\section{Introducción}

Desde 1994 existen en el ITESO (Instituto Tecnológico y de Estudios Superiores de Occidente, Guadalajara, México) una red de talleres artísticos estables y permanentes en las áreas de canto, literatura, artes plásticas y arte escénicas, todos se ofrecen a la comunidad universitaria. La Coordinación de estos talleres formaba parte de Promoción Cultural (PC), que a su vez pertenece al Centro de Servicios Comunitarios. Dentro de la Coordinación se encuentra el área de talleres y grupos artísticos. El objetivo del área de talleres y grupos artísticos consiste en apoyar el desarrollo de los miembros de la comunidad universitaria por medio de espacios para el conocimiento, el consumo, la práctica y el disfrute de las diferentes disciplinas artísticas. Dentro de los criterios pedagógicos del ITESO está considerar que el arte y la educación para el arte deben estar en la base de toda formación integral, "pues a través del arte, el ser humano se expresa y vive", y contar con un paradigma epistemológico basado en una triada: "experiencia, reflexión y acción", sugiriendo una multitud de caminos en los que los educadores podrían acompañar a sus alumnos y facilitarles el aprendizaje y la madurez.

El taller o aula de creación es una forma de organización "práctica y creadora del proceso de aprendizaje", un espacio interactivo donde se construyen conocimientos y se desarrollan capacidades y habilidades en un clima abierto, de confianza y libertad que estimula la realización individual y colectiva de los participantes. Se distinguen de las clases en las escuelas clásicas en que propician una interacción, una retroalimentación diferente entre el alumno y su profesor, porque se aprende haciendo. Es decir, el conocimiento no surge únicamente por la información y orientación que brinda el facilitador (instructor o especialista sobre el tema), sino también por la "participación activa de los integrantes del grupo" (López y Lee, 2011, p. 4). Aunque metodológicamente están establecidos los objetivos de investigación en esta área, la esencia sociocultural y los significados a nivel del grupo y la diversidad de manifestaciones culturales a partir del conocimiento etnológico y socio-histórico. 
La figura del artista-pedagogo es poco conocida en el contexto universitario. A pesar de que son frecuentes las referencias al "arte de enseñar" relacionadas con profesionales expertos en el manejo de situaciones de "incertidumbre, indeterminación, singularidad y conflicto", no es tan habitual identificar características propias del hacer docente en la intersección vital arte-universidad. En la figura del profesorado-artista se reconoce un saber docente que incorpora un pensamiento, unas estrategias y unas técnicas determinadas susceptibles a ser estudiadas (Montané, 2009b, p. 126).

El trabajo del artista-pedagogo responde a la búsqueda de escenarios complementarios donde los saberes, pedagógico y artístico, se encuentren y generen saberes didácticos movilizando la creatividad racional, intuitiva e integrativa que atiende a la perspectiva de Morin (2000, 2001), relacionada con el principio dialógico al entender los elementos antagónicos como complementarios. En estos escenarios pueden confluir diversas interpretaciones complementarias y diferentes lenguajes. Surge entonces una estructura emergente también responde a una preocupación intelectual por la forma, un desplazamiento hacia el "cómo" del pensamiento, más centrado en la significación y presentación de los fenómenos que en sus explicaciones causales para, finalmente, acabar recordando que el "reflejo en los espejos es múltiple y simultáneo", es decir, quedan interrogantes abiertos para poder continuar (Montané, 2009a, p. 119).

En las últimas décadas se ha hecho más explícito el carácter interdisciplinario de los estudios cualitativos, que son más asimilable a un espacio crítico de las ciencias sociales que a un tipo particular de teoría, metodología o filosofía (Schwandt, 2003). Si se asimila didáctica y arte, y al mismo tiempo se entiende el arte como organismo vivo (Vygotsky, 2005), generando una didáctica que se ha denominado "apreciativa y/o generativa”, con una serie de acciones específicas (Jove, 2002, pp. 25-26):

1) Visualiza la situación didáctica como posibilidad, el punto de partida no es el déficit del alumnado sino la "potencia de sus posibilidades".

2) Parte de una "mirada apreciativa", positiva, del sujeto.

3) Trasciende un pensamiento crítico y lo convierte en "generativo". Busca reinventar las posibilidades para el cambio y la acción.

4) Se trata de una situación más "colaborativa" que dirigida.

5) Trabaja con distintos lenguajes: verbales, no verbales, corporales, visuales, tecnológicos...

6) Crea con el imaginario y la visualización. 
7) Es transdisciplinar y compleja.

Tras la ausencia de estudios sobre el impacto real que producen los talleres artísticos estables y permanentes en Educación Superior, y ante la necesidad de legitimar y hacer crecer la tarea que Promoción Cultural del ITESO llevaba haciendo; nace un proyecto de investigación que pretende recuperar la experiencia vivida, y objetivar los resultados de estas actividades artísticas dentro de la formación de las personas participantes en los talleres. Inicialmente se pretende responder a dos objetivos: por un lado recuperar documentalmente la experiencia y divulgarla, y por otro generar espacios de reflexión y discusión sobre el estado del arte en el entorno.

\section{Metodología}

El trabajo de campo de la investigación se extiende a lo largo de tres años, dividido en dos fases:

\section{$1^{\mathrm{a}}$ fase. Estudio inicial}

Se parte de una recuperación sistematizada de la experiencia de los participantes, y se elabora un instrumento de evaluación que permitiera retroalimentar el proyecto de talleres de Promoción Cultural (PC).

La muestra estuvo integrada por cinco personas de cada taller, incluido su coordinador. Se realizaron dos sesiones y se obtuvo la respuesta de alrededor de cincuenta personas. La selección de la muestra fue al azar, con dos criterios de inclusión: "llevar un año o más de antigüedad en el taller, y ser residente de la ciudad de Guadalajara".

La metodología estuvo centrada en la exploración y descripción de motivos, significados, grado de satisfacción y desarrollo de emociones del impacto humano y formativo de los distintos talleres artísticos como proceso y red de aprendizaje vivencial. Se pretendía abarcar la experiencia en su totalidad, obteniendo las variables en función de la evolución del propio diseño. Además se aspiraba a adaptar escalas de motivación y satisfacción, así como cuestionarios ya validados y estandarizados sobre el impacto emocional. Se elaboró una encuesta-sondeo conformada de siete preguntas. El siguiente paso consistió en el análisis cualitativo de las respuestas obtenidas, con lo que se establecieron categorías de análisis basadas en las teorías del aprendizaje. En la muestra había dos tipos de indicadores de observación: indicadores internos, o información referida y observada por las personas que participan directamente en la experiencia de los talleres artísticos, e indicadores externos, o información referida por las personas cercanas a quienes participan en los talleres, que conocen a las personas desde antes de pertenecer al taller. 
$2^{\mathrm{a}}$ fase. Contraste y continuidad

Los resultados de la primera etapa sirven como punto de partida en la segunda. Con base en los resultados globales de la encuesta-piloto, se pretende ahondar en la experiencia específica de cada taller y conocer de manera indirecta el impacto en el contexto personal de los participantes (familia, escuela, trabajo). Se parte de la idea de que el contexto de cada persona se ve reflejado en su interacción con sus compañeros de taller: la manera de relacionarse, cómo el proceso de expresión artística permite sensibilizarse y mostrar aspectos de su vida personal. La información que se presenta en los apartados de crecimiento personal y crecimiento en grupo fue rescatada de 57 encuestas a participantes de los distintos talleres artísticos, y los apartados de conclusiones y detección de necesidades están enriquecidos por entrevistas a participantes de los talleres y a maestros; dos registros de observación de sesiones completas en cada taller, tres registros de observación de sesiones de retroalimentación y dos de actividades.

\section{Resultados del proyecto}

Exponemos una síntesis de los hallazgos en la siguiente tabla que divide en tres grandes criterios de observación el impacto en los participantes de cada uno de los talleres: motivación inicial, impacto (compuesto por tres áreas: desarrollo personal, social y estético) y necesidades (demandas expresadas sobre organización, espacio y crítica general). 


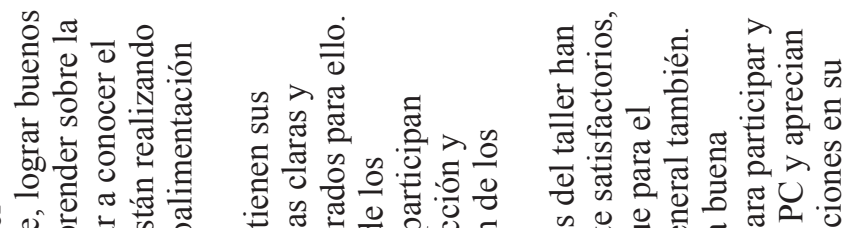

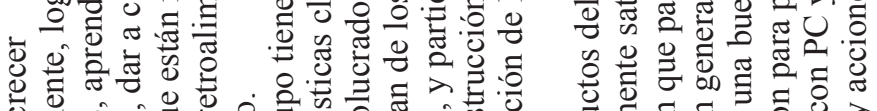

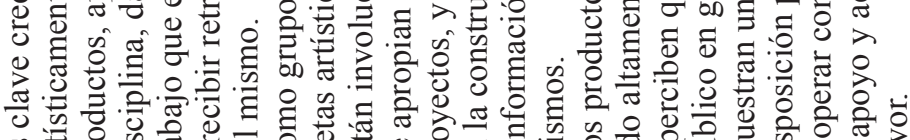

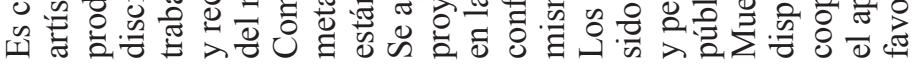

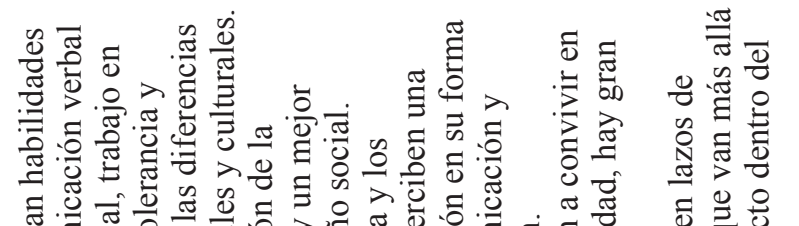

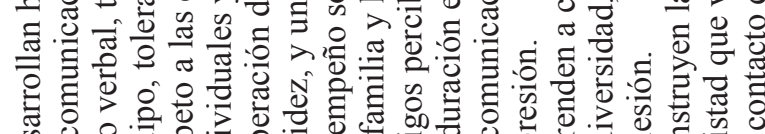

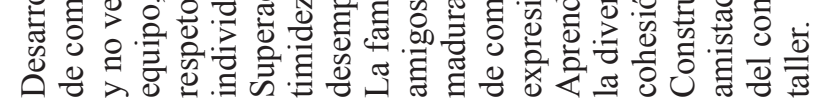

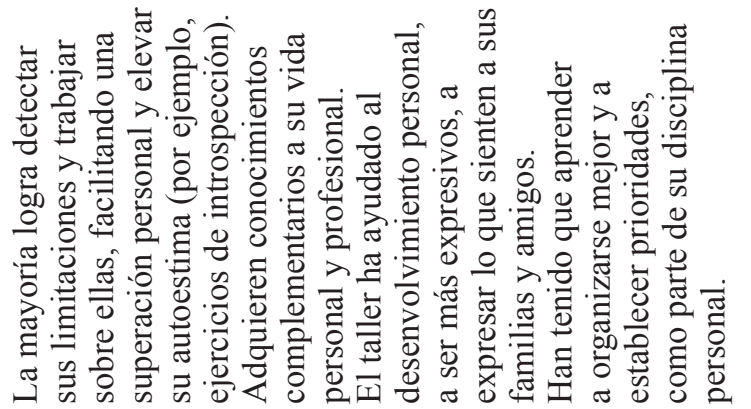

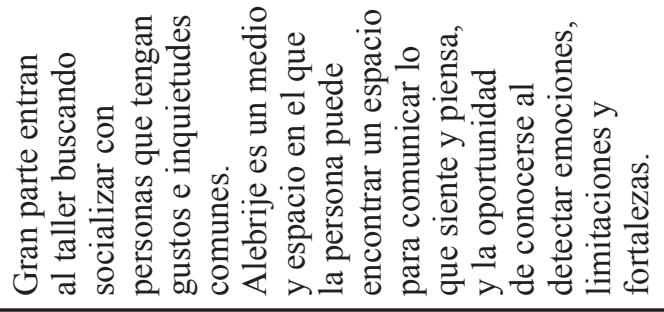

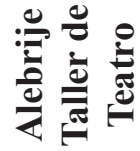




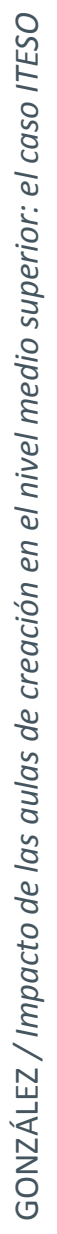

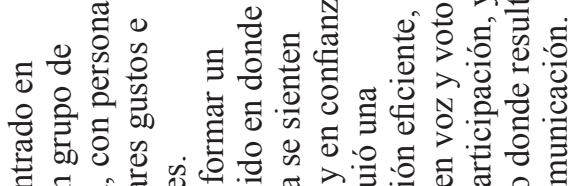

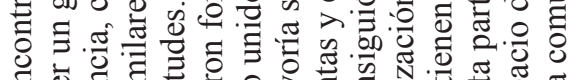

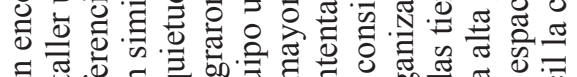

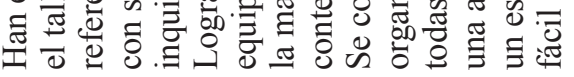

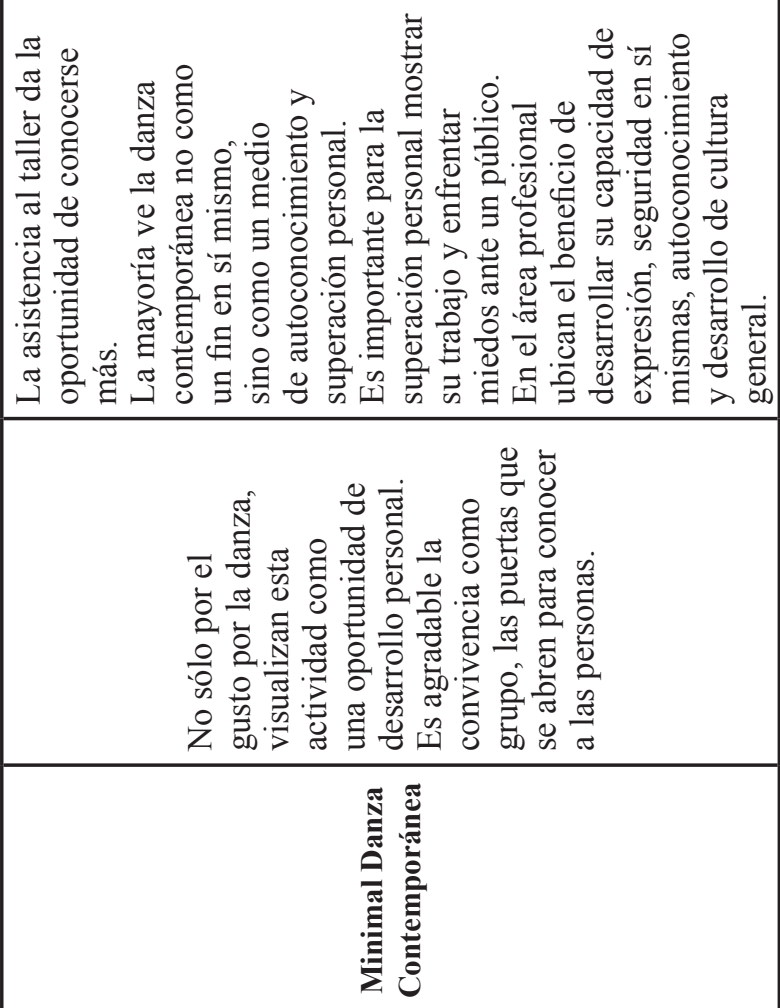




\section{Discusión de resultados}

Cada taller tenía unas diferencias, necesidades y aspectos técnicos específicos; y compartían unas necesidades generales, visiones y vivencias comunes; destacando todos los participantes la figura de sus maestros, con unas funciones concretas, y una vocación que las diferencia del común de los docentes de escuela formal, empezando por una mayor relevancia e impacto por su calidad y grado de influencia.

Así mismo, se detecta una especial relevancia de la tarea de formación de públicos y la obtención de fondos. El nivel de creatividad también se extiende para concebir nuevas maneras de ofertar el hecho cultural y artístico, pues los medios de difusión tradicionales ya no resultan suficientes ante la saturación informativa que vivimos; es un hecho que, en los últimos años, la masificación de la cultura ha llevado a consumir lo que los medios de comunicación expresan a partir de lo visual y lo auditivo, entre otros. La difusión de la cultura debe ser también "un hecho creativo y formativo que conjugue la estética y la imaginación" (Vygotsky, 2004, pp. 3-4), unido a buscar espacios alternativos, ya que los institucionales están saturados.

En el desarrollo de la investigación hay una serie de puntos, detectados en distintas líneas de investigación, que pudieran favorecer y apoyar un "enfoque proactivo de la creatividad" (Root-Bernstein, 2002):

- Autoeficacia creativa: la confianza en la capacidad y competencia de uno mismo para ser creativamente productivo es un gran estímulo. La autoeficacia creativa fomenta los estilos de pensamiento que posee la gente creativa, como la curiosidad o la capacidad de asumir riesgos intelectuales (Beghetto, 2006).

- Libertad y seguridad psicológicas: para mantener esta autoeficacia creativa debe haber un ambiente de apoyo. Siempre cabe la posibilidad de equivocarse, pero un entorno propicio para la creatividad otorgará a los estudiantes la libertad de asumir riesgos y la seguridad de que los errores no tengan consecuencias negativas. Los espacios de formación creativa deben de ser un lugar seguro en el que equivocarse, algo a tomar nota en escuelas y universidades. Una institución educativa creativa es incompatible con el miedo a la autoridad, especialmente cuando es al profesor a quien se considera autoridad (Romo, 2013, p. 7).

- Aprendizaje o resolución de problemas lúdicos: además del placer y la diversión, asociar el juego con el aprendizaje tiene consecuencias muy positivas para el desarrollo evolutivo (Nickerson, 2010). Por desgracia, existe una fuerte barrera cultural en contra, resumida dramáticamente en el refrán: «la letra, con sangre entra», que aún puede resistirse a desaparecer. Es importante fijar las fuertes conexiones que vinculan el juego con la creatividad y el mundo de posibilidades que abre (Del Río y Álvarez, 2007, pp. 113-132). 
- Transmisión de conocimientos: la revisión de distintos trabajos apuntan a que la función psicológica más significativa de la creatividad es el pensamiento analítico, o capacidad para conectar ideas muy diferentes entre sí y de hacer "asociaciones remotas" (Romo, 2013). Se enfatiza un enfoque interdisciplinar, promoviendo el uso de analogías que simplifican el aprendizaje de nuevos conceptos (Fairweather y Cramond, 2010).

- Perspectiva estética y mirada artística: este área de conocimiento viene concretada por los saberes, percepciones y creencias previas de los participantes sobre la perspectiva artística, sus "teorías implícitas" (Sternberg, 1985); desde la comprensión de la educación como un arte, hasta una noción amplia y abierta de creatividad. Se alude a las aportaciones del arte y a la educación desde el arte; al rol social de los artistas y a su impacto social (Montané, 2009b, p. 121). Se establecen las relaciones arte-educación siempre desde su perspectiva y situación, incluyendo una dimensión terapéutica.

- Síntesis educativa: una de las principales trabas en el proceso de enseñanza y aprendizaje creativo es la "compartimentación del conocimiento" (Romo, 2013, p. 8). Tras un proceso de especialización de disciplinas científicas que comenzó con la revolución copernicana, estamos volviendo a la reunificación de áreas del conocimiento: vivimos en la "era de la interdisciplinaridad". Cropley (2001, p. 17) afirmó que el estudiante creativo ideal en la enseñanza media y superior posee una «visión de helicóptero» que implica el dominio de un campo especial y la percepción de interconexiones entre campos diferentes (Root-Bernstein, 2002, pp. 368-369).

- Apertura a una retroalimentación crítica: las actividades frente a un público, presentaciones, exposiciones, muestras, actuaciones, intervenciones, abren un mundo de posibilidades a cada participante. La motivación extrínseca basada en resultados académicos vividos como obligación, ha tornado motivación intrínseca y de logro personal expuesta por iniciativa propia ante una audiencia externa a la academia.

\section{Conclusiones}

En Iteso surgió la propuesta de hacer una recuperación sistematizada de las experiencias significativas de aprendizaje, y a la vez lograr un instrumento de evaluación que permitiera retroalimentar el proyecto con sus distintos talleres. Desde el inicio los resultados indicaron que los participantes percibían como objetivos generales "aprender a aprender" y "generar y mantener espacios de expresión". Por lo tanto, no sólo esperaban aprender técnicas concretas, sino también disfrutar de un espacio para participar activamente. 
La motivación intrínseca para participar en este tipo de talleres, lleva a que cada integrante pueda contribuir al descubrimiento de nuevos aspectos de su potencial, y al desarrollo de varias dimensiones de su autoconcepto. Conocerse y valorarse más, al mismo tiempo que sentirse capaz de compartir su potencial con otros (Gardner, 1999). Desde Dewey (1949) se reconoce la importancia clave del aprendizaje en el medio real, la experiencia directa. Fuera del medio se puede reflexionar y tomar los primeros contactos teóricos con un aprendizaje, pero el aprendizaje efectivo debe realizarse en contacto directo con la realidad donde y sobre la que se haya de actuar.

En cada uno de los talleres analizados se encontraron semejanzas en la manera de conducir la experiencia, en todos subyace un proceso común. En primera instancia se parte de lo básico de cada disciplina, su campo de actuación, a la vez que se va contextualizando e introduciendo a los participantes en el taller, procurando generar un clima de grupo que permita disfrutar la experiencia. En una segunda fase hay un ejercicio continuo a partir de una práctica cotidiana, de rutina y discusión, aprendiendo la jerga del ámbito, a la vez que van aumentando los conocimientos técnicos y confiriéndolos un sentido. En tercer lugar, va emergiendo la fase del aprendizaje y la imaginación creativa en la que se invita a crear siguiendo unas directrices mínimas ya conocidas, que atienden a la necesidad de aportación antes mencionada. Este proceso ha sido observado y confirmado en distintas experiencias de talleres, aulas y grupos en Latinoamérica y Europa.

Dejamos abiertas algunas cuestiones para siguientes trabajos: ¿qué papel tiene la educación artística en el proceso de formación de las personas?, ¿pueden los docentes valerse de actividades artísticas como métodos didácticos o de apoyo para sus clases académicas?, ¿qué contenidos y procesos de enseñanza y aprendizaje favorecen el ejercicio profesional de un artista?

\section{Referencias bibliográficas}

Beghetto, R. (2006). Creative self-efficacy: Correlates in middle and secondary students. Creative Research Journal, 18, 447-457.

Beghetto, R. y Kaufmann, J. (2010). Nurturing Creativity in the Classroom. Cambridge UniversityPress.

Bisquerra, R. (2012). Las emociones en el aprendizaje. En Competencias de profesor de ELE. III Encuentros Comillas, 10-12 Noviembre de 2011. Fundación Comillas y MEC.

Csikszentmihalyi, M. (1997). Creativity: Flow and the Psychology of Discovery and Invention. New York: Harper Perennial.

Csikszentmihalyi, M. (1998). Finding Flow: The Psychology of Engagement With Everyday Life. Basic Books. 
De Bono, E. (1991). El pensamiento lateral. Barcelona: Paidós Ibérica.

Del Río, P. y Álvarez, A. (2007). Escritos sobre arte y educación creativa de Lev S. Vygotski. Madrid: Fundación Infancia y Aprendizaje.

Denzin, N., y Lincoln, S. (2003). Introduction. The discipline and Practice of Qualitative Research. En: Denzin, N., y Lincoln, S. Collecting and Interpreting Qualitative Materials. London: Sage.

Dewey, J. (1949). El arte como experiencia. México: Fondo de Cultura Económica.

Díaz de León, M. L. y Cruz, L. (2001). Recuperación de la experiencia en el proyecto del área de talleres, $2^{a}$ etapa. ITESO: México. (Documento inédito).

Díaz de León, M. L. y González, J. (2000). Recuperación de la experiencia en el proyecto del área de talleres, $1^{a}$ etapa. ITESO: México. (Documento inédito).

Fairweather, E. y Cramond, B. (2010). Infusing creative and critical thinking into the curriculum together. En Beghetto, R. y Kaufmann, J. Nurturing creativity in the classroom. Cambridge UniversityPress.

Fernández-Berrocal, P., Ruiz-Aranda, D., Extremera, N., Cabello, R. (2009). ¿Es posible crear una escuela emocionalmente inteligente?, 37-55. En Augusto, J.M. (coord.) Estudios en elámbito de la inteligencia emocional. Jaén: Universidad de Jaén.

Fisher, E. (1979). La necesidad del arte. Barcelona: Icaria.

Gardner, H. (1994). Educación artística y desarrollo humano. Buenos Aires: Paidos Educador.

Gardner, H. (1999). Inteligencias múltiples. La teoría en la práctica. Barcelona: Paidós.

Hennessey, B. (2010). Intrinsic motivation and creativity in the classroom: Have we come full circle? En Beghetto, R. y Kaufmann, J. Nurturing creativity in the classroom. Cambridge UniversityPress.

Jové, J. J. (2002). Arte, psicología y educación. Madrid: Machado Libros.

Kaufman, J. y Sternberg, R. (1999). The Cambridge Handbook of Creativity. Cambridge.

Kutchins, H. y Kirk, S. A. (1997). Making Us Crazy. DSM: The Psychiatric Bible and the Creation of Mental Disorders. New York/London: The Free Press.

Laferrière, G. (2000). Sesiones de trabajo con los pedagogos de hoy. Ciudad Real: Naque. 
López de la Llave, A. y Pérez-Llantada, M. C. (2006). Psicología para Intérpretes Artísticos. Madrid: Thomson Paraninfo.

López, O. y Lee, M. (2011). Los talleres de apreciación y creación artística para preservación y promoción de la cultura. Contribuciones a las Ciencias Sociales, Julio 2011. Recuperado el 2/05/2014 en http://www.eumed.net/rev/cccss/13/lall.htm.

Maffesoli, M. (1997). Elogio de la razón sensible: una visión intuitiva del mundo contemporáneo. Barcelona: Paidós.

Montané, A. (2009a). Universidad, complejidad y arte: texturas educativas. En A. Guillaumin, y O. Ochoa, Hacia otra educación. Miradas desde la complejidad. Xalapa-México: Arana.

Montané, A. (2009b). Profesores-artistas y didáctica generativa: Experiencias educativas en la universidad [En línia] REIRE: Revista d'Innovació i Recerca en Educació, 2, 117-129. Consultado el 22/10/2013 en http://www.raco.cat/index.php/ REIRE.

Moreno, S. (2002). Aportes de la educación artística a la formación profesional de la persona. México: ITESO. (Documento inédito).

Moreno, S. (2003). Recuperación del proceso formativo de los participantes de los talleres artísticos. México: ITESO. (Documento inédito).

Morín, E. (2001). Los siete saberes necesarios para la educación del futuro. Barcelona: Paidós.

Romo, M. (2013). Algunas investigaciones sobre el impacto de la creatividad en el ámbito educativo. Fundación Marcelino Botín. Consultado el 22/11/2013 en http:// www.fundacionbotin.org/innovation-education-platform_good-morning-creativity. htm

Root-Bernstein, R. M. (2002). El secreto de la creatividad. Barcelona: Kairós.

Rose, N. (1998). Inventing Ourselves Psychology, Power and Personhood. Cambridge: Cambridge UniversityPress.

Schön, D. A. (1998). El profesional reflexivo: cómo piensan los profesionales cuando actúan. Barcelona: Paidós.

Schwandt, T. (2003). Three epistemological stances for qualitative inquiry: interpretivism, hermeneutics, and social constructionism. En K.D. Norman, \& Y. Lincoln, Collecting and Interpreting Qualitative Materials. Londres: Sage.

Steinem, G. (1996). Revolución desde dentro. Madrid: Anagrama. 
Sternberg, R. J. y Davidson, M. (2005). (Eds). Conceptions of Giftedness. CambridgeUniversity Press.

Sternberg, R. J. (1986). A triangular theory of love. Psychological Review, 93, 119 135 .

Sternberg, R. J. (1997). A Triarchic View of Giftedness: Theory and Practice. In N. Coleangelo\& G. A. Davis (Eds.), Handbook of Gifted Education, 43-53. Boston: Allyn and Bacon.

Sternberg, R. J. (1999). The theory of successful intelligence. Psychology Journal: practice and Research, 55 (3), 139-154.

Sternberg, R.J. (1985). Implicit theories of intelligence, creativity and wisdom. Journal of Pesonality and Social Psychology, 49, 607-627.

Vygotsky, L. S. (2004). Imagination and Creativity in Childhood. Journal of Russian and East European Psychology 42, 7-97. (Original 1931).

Vygotsky, L. S. (1990). La imaginación y el arte en la infancia. Barcelona: Akal (Original 1932).

Vygotsky, L. S. (2005). Psicología del arte. México: Fontanamara. (Original 1925). 


\section{ANEXO}

\section{Instrumentos Investigación}

Anexo 1. Entrevista de sondeo para maestros y alumnos. $1^{\text {a }}$ fase

1. ¿A qué pretenden responder los talleres?

2. ¿A qué pretende responder tu taller?

3. ¿Qué contenidos mínimos debe tener el taller?

4. ¿Cómo es el método que desarrollas para impartir tu taller?

5. ¿Cuáles son las actitudes que has desarrollado en tu taller?/ ¿Cuáles son las actitudes que has desarrollado en el taller?

6. ¿Cuál es el principal objetivo de tu taller?

7. ¿Con qué obstáculos te han encontrado para el desarrollo del taller con respecto a los objetivos planteados?

8. ¿Qué crees que aporta a la formación académica de los alumnos?/ ¿Qué te aporta el taller en tu formación académica?

9. ¿Qué crees que empuja a los alumnos a pertenecer al taller? / ¿Qué te empuja a pertenecer al taller?

10. ¿Cuáles serían los mínimos aprendizajes, valores o experiencias que te gustaría dejar en los alumnos?/ ¿Qué te ha dejado el taller?

11. ¿Qué cambiarías de tu taller?

12. ¿Qué crees que no deberíamos olvidar al hacer este estudio? 
Anexo 2. Cuestionario de Evaluación $2^{\mathrm{a}}$ fase.

PROPUESTA DE INSTRUMENTO DE EVALUACIÓN PARA PARTICIPANTES

Nombre: Taller:

A cada afirmación responde con la letra que corresponda al rango de porcentaje en que se logró este semestre. Los rangos de porcentaje son los siguientes:
$\mathrm{A}=0-25 \%$
$\mathrm{B}=26-50 \%$
$\mathrm{C}=51-75 \%$
$\mathrm{D}=76-100 \%$

Por ejemplo: $\quad$ - La puntualidad A

1.- Aprendizaje de técnicas

2.- Trabajo en equipo

4.- Claridad en el objetivo de talleres

5.- Conveniencia del horario

6.- Puntualidad

7.- Grado de compromiso

8.- Difusión de la existencia de los talleres

9.- Difusión de las presentaciones

10.- Calidad de las presentaciones

11.- Crecimiento personal

12.- Crecimiento profesional

13.- Desarrollo de habilidades artísticas

14.- Asistencia a eventos artísticos

15.- Apoyo entre talleres

16.- Comunicación entre talleres

17.- Integración grupal

18.- Condiciones de trabajo en el taller

19.- Cumplimiento de aspiraciones personales

20.- Satisfacción con trabajo del coordinador

21.- Relación con Coordinador 